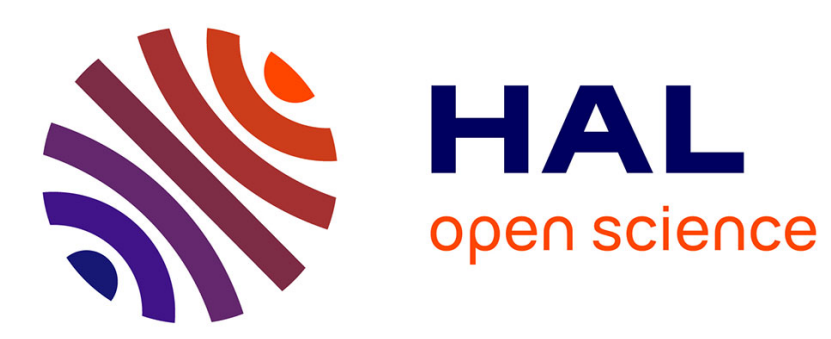

\title{
Recherche d'un couple frigorigène adapté à la réfrigération solaire en zone tropicale
}

\author{
J. Fléchon, F. Machizaud
}

\section{To cite this version:}

J. Fléchon, F. Machizaud. Recherche d'un couple frigorigène adapté à la réfrigération solaire en zone tropicale. Revue de Physique Appliquée, 1979, 14 (1), pp.97-105. 10.1051/rphysap:0197900140109700 . jpa-00244596

\section{HAL Id: jpa-00244596 https://hal.science/jpa-00244596}

Submitted on 1 Jan 1979

HAL is a multi-disciplinary open access archive for the deposit and dissemination of scientific research documents, whether they are published or not. The documents may come from teaching and research institutions in France or abroad, or from public or private research centers.
L'archive ouverte pluridisciplinaire HAL, est destinée au dépôt et à la diffusion de documents scientifiques de niveau recherche, publiés ou non, émanant des établissements d'enseignement et de recherche français ou étrangers, des laboratoires publics ou privés. 


\title{
Recherche d'un couple frigorigène adapté à la réfrigération solaire en zone tropicale
}

\author{
J. Fléchon et F. Machizaud \\ Laboratoire de Physique des Dépôts Métalliques, Université de Nancy I, \\ C.O. 140, 54037 Nancy Cedex, France \\ (Reçu le 29 juin 1978, révisé le 2 octobre 1978, accepté le 2 octobre 1978)
}

\begin{abstract}
Résumé. - On examine les possibilités de réfrigération solaire par utilisation de capteurs plans associés à un bouilleur contenant un mélange binaire $\mathrm{NH}_{3}, \mathrm{H}_{2} \mathrm{O}$ ou $\mathrm{NH}_{3}, \mathrm{NaSCN}$. On montre, à partir des diagrammes $1 / T$, Log $P$ qu'en zone tropicale, l'absorption sèche $\left(\mathrm{CaCl}_{2}, 8 \mathrm{NH}_{3}\right)$ semble la solution la meilleure.
\end{abstract}

Abstract. - The possibilities of solar refrigeration by associated with a flat plate collector a and a boiler containing a binary solution $\mathrm{NH}_{3}, \mathrm{H}_{2} \mathrm{O}$ or $\mathrm{NH}_{3}, \mathrm{NaSCN}$ are examined. From the diagrams $1 / T, \log P$ it is shown that in tropical zone, the solid absorption $\left(\mathrm{CaCl}_{2}, 8 \mathrm{NH}_{3}\right)$ seems to be the better solution.

Une étude systématique des insolateurs plans $[1,2,3]$ nous a permis de définir une température limite d'équilibre pour un collecteur soumis à un rayonnement global donné dans des conditions réelles définies. Des considérations cinétiques [4] ont montré que la durée d'insolation impose pour le capteur la définition d'un temps de relaxation compatible avec la nécessité d'atteindre au cours de l'exposition au soleil une température suffisante dans un délai assez bref afin de le rendre utilisable.

L'examen de ces divers paramètres conduit, en considérant les facteurs climatiques d'un lieu donné, à une optimisation du récepteur devant servir dans nos régions pour le chauffage, et pour la réfrigération dans les pays tropicaux.

1. But. - Le but du présent texte est de montrer que les réfrigérateurs solaires̀ à absorption associés à un capteur plan ne peuvent valablement fonctionner que dans des limites rigoureusement définies.

Par ailleurs des appréciations divergentes [5, 6] étant formulées sur les performances comparées des mélanges binaires ammoniac-eau, ammoniac-sulfocyanure de sodium il convient de faire le point à partir de données thermodynamiques indiscutables.

En effet Swartman [5] traitant dans des conditions comparables d'irradiation solaire les mélanges $\mathrm{NH}_{3}-\mathrm{H}_{2} \mathrm{O}$ et $\mathrm{NH}_{3}-\mathrm{NaSCN}$ obtient des coefficients de performance solaire qui varient

- dans le premier cas de 0,05 à 0,14 ,

- dans le second cas de 0,11 à 0,27 ,

alors que Eggers-Lura [6] trouve pour $120^{\circ}$ au bouilleur des coefficients de 0,10 avec le binaire $\mathrm{NH}_{3}-\mathrm{H}_{2} \mathrm{O}$ et 0,08 avec $\mathrm{NH}_{3}-\mathrm{NaSCN}$.
Nous désirons réaliser un appareil fonctionnant sans intervention extérieure et sans mécanisme mobile. consommateur d'énergie. Il s'agit d'utiliser, sans intermédiaire, les calories captées par un insolateur plan à la vaporisation d'un corps pur qui, condensé sous pression, s'évaporera lorsque le milieu qui l'a libéré sous l'action de la chaleur sera susceptible de le réabsorber lorsque sa température sera plus basse.

Ces conditions imposent de retenir le système à absorption intermittente qui se présente sous deux formes :

- absorption humide,

- absorption sèche.

Le corps pur fluide réfrigérant est généralement l'ammoniac. L'absorption humide correspond à un phénomène physique de dissolution simple de l'ammoniac par exemple dans l'eau, le nitrate de lithium $\mathrm{LiNO}_{3}$ ou le thiocyanate de sodium NaSCN. L'absorption sèche repose sur un phénomène chimique : la formation et la dissociation d'un ammoniacate solide à partir d'halogénures d'alcalins et d'alcalinoterreux parmi lesquels les plus couramment utilisés sont le chlorure de calcium $\mathrm{CaCl}_{2}$ et le chlorure de strontium $\mathrm{SrCl}_{2}$.

Ces deux processus sont caractérisés par des propriétés particulières les rendant plus ou moins utilisables en réfrigération et climatisation. Leur emploi pratique intéresse essentiellement les pays tropicaux en voie de développement dans les régions privées d'énergie électrique mais où l'énergie solaire échauffera suffisamment l'absorbant pour lui faire libérer le fluide frigorigène qui, condensé, permettra durant la nuit de produire du froid par évaporation. 
Or, si ce phénomène est simple, puisqu'il utilise les chaleurs latentes de vaporisation, de dissolution et de dissociation, il semble d'après Eggers-Luna et al. [6] que malgré de très nombreuses études au laboratoire aucune réalisation pratique industriellement utilisable à partir de l'énergie solaire n'a encore été mise en chantier. Ceci signifie qu'au-delà des principes, certains paramètres apparemment mineurs peuvent devenir déterminants. Telle est par exemple, dans les conditions réelles d'emploi, la vitesse de fixation de l'ammoniac durant la nuit. Elle est fonction, en particulier, dans ces systèmes quasi réversibles, des taux de transfert thermique. Si elle est trop lente, le rendement est dérisoire et l'appareil ne peut rendre aucun service.

Cependant, il est possible de jouer sur cette vitesse par des dispositifs augmentant l'importance des surfaces gaz-absorbant et assurant l'homogénéisation de la solution de façon à éviter les gradients de concentration qui tendent à réduire l'absorption.

2. Principe de l'étude. - Schématiquement le réfrigérateur solaire à absorption intermittente comprend un générateur ou bouilleur qui contient le binaire ammoniac-eau - par exemple - solidaire d'un collecteur plan et communiquant par l'intermédiaire d'un condenseur avec un évaporateur renfermant l'ammoniac liquide. La pression régnant dans les deux enceintes est donc la même lorsqu'une fraction du système ne subit pas d'évolution importante. Or tout échange énergétique quotidien avec le rayonnement solaire se situe entre la période la plus chaude de la journée et l'ambiance la plus froide de la nuit.

Les performances théoriques maximales de l'appareil seront déterminées à partir de deux équilibres extrêmes :

- l'un, vers 15 heures, lorsque sera atteinte la température $T_{\mathrm{Mb}}$ la plus élevée du bouilleur chauffé par le collecteur plan : à cet instant la concentration en ammoniac du binaire est la plus faible $C_{\mathrm{m}}$, la pression $P_{\mathrm{M}}$ qu'il subit est au maximum celle qui règne dans le condenseur c'est-à-dire la pression définissant l'équilibre liquide-vapeur de $\mathrm{NH}_{3}$ à la température $T_{\mathrm{Mc}}$ de la paroi du condenseur,

- l'autre, au lever du soleil, après l'évaporation de l'ammoniac durant la nuit par suite de l'existence d'une dépression au-dessus du binaire dès que la température du bouilleur privé d'énergie rayonnante se sera abaissée pour atteindre la température $T_{\mathrm{mb}}$ qui pourra être égale ou légèrement supérieure à l'ambiante.

A cet instant la pression $P_{\mathrm{m}}$ est la même dans l'évaporateur et le bouilleur. $P_{\mathrm{m}}$ définit l'équilibre liquidevapeur de $\mathrm{NH}_{3}$ à la température $T_{\text {me }}$ de la paroi de l'évaporateur ainsi que celui du binaire de concentration la plus forte $C_{\mathrm{M}}$ à la température $T_{\mathrm{mb}}$.

Ainsi $P_{\mathrm{M}}$ est directement lié à $T_{\mathrm{Mb}}, T_{\mathrm{Mc}}$ et $C_{\mathrm{m}}$ de même que $P_{\mathrm{m}}$ est fonction de $T_{\mathrm{mb}}, T_{\mathrm{me}}$ et $C_{\mathrm{M}}$.
Il est possible de définir l'espérance théorique maximale permise par l'utilisation d'un absorbant particulier dans des conditions climatiques données. Il suffit, en effet, de tracer pour chaque système binaire étudié dans le plan $(P, T)$ le réseau des courbes expérimentales obtenu à partir des concentrations utilisables en réfrigération solaire.

Or dans la représentation $\log P, 1 / T$ utilisée par les techniciens du froid [7] pour le couple $\mathrm{NH}_{3}-\mathrm{H}_{2} \mathrm{O}$ le diagramme type de Bosnjakovic et Wucherer [7] est particulièrement simple puisqu'il est formé d'un faisceau de droites.

3. Les diagrammes $(1 / T, \log P)$. - Nous nous proposons de travailler, sur ce diagramme dont l'emploi est d'une pratique courante en réfrigération industrielle pour définir les possibilités du binaire $\left(\mathrm{NH}_{3}, \mathrm{H}_{2} \mathrm{O}\right)$ puis de construire à partir de l'expérience un diagramme semblable pour d'autres couples afin de comparer les caractères de chacun d'eux. Nous les limiterons au domaine de température intéressant pour la réfrigération et permis par l'intensité du rayonnement solaire non concentré soit $-10^{\circ} \mathrm{C}$, $+140^{\circ} \mathrm{C}$.

3. 1 LE COUPle $\mathrm{NH}_{3}-\mathrm{H}_{2} \mathrm{O}$ (Fig. 1). - Supposons que le bouilleur soit porté à $T_{\mathrm{Mb}}=120^{\circ} \mathrm{C}$ (point $\mathrm{E}_{1}$ ) le condenseur étant maintenu à $T_{\mathrm{MC}}=30^{\circ} \mathrm{C}$ (point $\left.\mathrm{A}_{1}\right)$. Nous connaîtrons la concentration minimale à l'équilibre du binaire de la manière suivante : sur l'axe des températures partons de $A_{1}$, suivons la verticale issue de $A_{1}$ elle coupe en $B_{1}$ la droite définissant l'équilibre liquide-vapeur de $\mathrm{NH}_{3}$. $\mathrm{B}_{1}$ définit la pression régnant dans le condenseur et le bouilleur porté à $120^{\circ} \mathrm{C}$ : point $\mathrm{D}_{1}$. Nous connaissons ainsi la concentration en ammoniac $C_{\mathrm{m}}$ de la solution pauvre soit dans l'exemple cité : $25 \%$.

Au lever du jour si $T_{\mathrm{mb}}=30^{\circ}$ au bouilleur dont la solution s'est enrichie au cours de l'évaporation

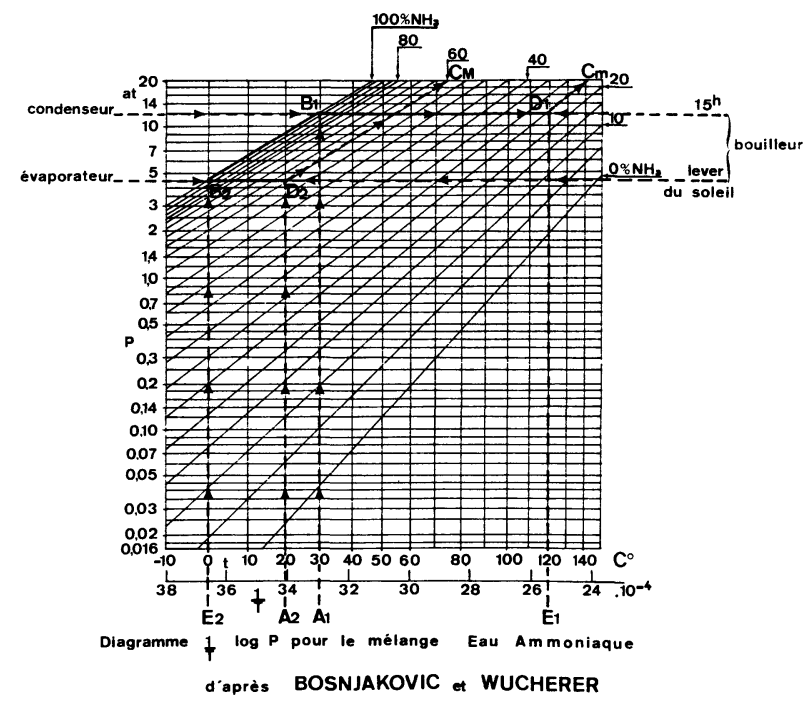

Fig. 1. - Diagramme 1/T, $\log P$ pour le mélange $\mathrm{H}_{2} \mathrm{O}, \mathrm{NH}_{3}$. [Diagram $1 / T, \log P$ for the mixture $\mathrm{H}_{2} \mathrm{O}, \mathrm{NH}_{3}$.] 
nocturne et $T_{\mathrm{me}}=0^{\circ} \mathrm{C}$ à l'évaporateur, nous pourrons connaître de même la concentration $C_{\mathrm{M}} \mathrm{du}$ mélange. En partant de $E_{2}$ et en suivant le trajet $B_{2}$, $\mathrm{D}_{2}$ qui est à la verticale de $\mathrm{A}_{2}$ : ici $C_{\mathrm{M}}=60 \%$. Nous en déduisons immédiatement $C_{\mathrm{M}}-C_{\mathrm{m}}=\Delta C \%=35 \%$ qui représente le taux d'ammoniac utilisable pour la réfrigération. En fait, nous verrons que $C_{\mathrm{m}}$ et $C_{\mathrm{M}}$ correspondent à des masses différentes de solution et qu'un calcul complémentaire s'impose.

3.2 Le COUPLE $\mathrm{NH}_{3}-\mathrm{NaSCN}$. - G. C. Blytas et F. Daniels [8] examinent les écarts à la loi de Raoult de différents sels en solution dans l'ammoniac liquide. Ils précisent que jusqu'en 1962 aucune mesure n'a été faite sur le couple $\mathrm{NH}_{3}-\mathrm{NaSCN}$. Au cours de nombreuses expériences ils définissent quelques caractères physicochimiques de la solution : solubilité, densité, pression de vapeur, conductivité thermique, chaleur de dissolution et capacité calorifique. L'ensemble de leurs résultats permet de tracer le réseau $(1 / T, \log P)$ pour les concentrations applicables à la réfrigération solaire (Fig. 2), S. L. Sargent et W. A. Beckman [16] étudient les performances théoriques d'un cycle intermittent utilisant ce couple.

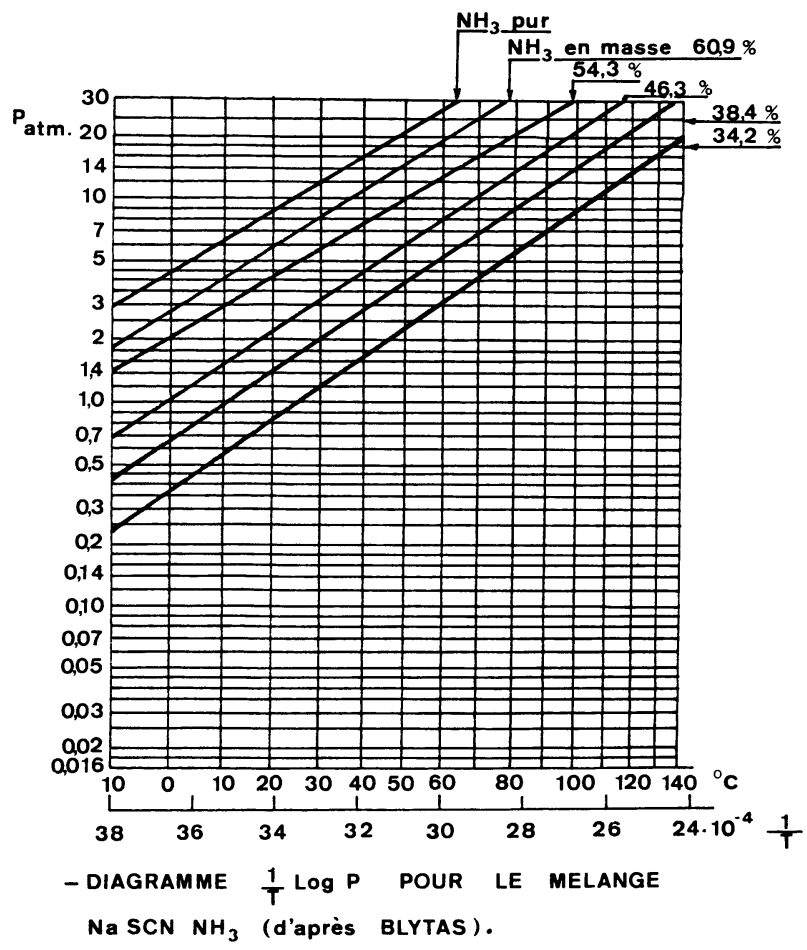

Fig. 2. - Diagramme 1/T, Log $P$ pour le mélange $\mathrm{NaSCN}, \mathrm{NH}_{3}$.

[Diagram $1 / T, \log P$ for the mixture $\mathrm{NaSCN}, \mathrm{NH}_{3}$.]

En examinant le réseau de la figure 2 nous procédons au même traitement qu'en 3.1 à partir de conditions imposées par le climat et l'énergie solaire incidente. Nous pourrons alors comparer les possibilités des deux couples en leur fixant les mêmes servitudes.
4. La réfrigération en zone tropicale. - 4.1 CLIMATOLOGIE. - Nous référant aux statistiques relevées en 20 ans par la station météorologique de Bamako (Mali) nous notons que la saison chaude a lieu entre mars et juin avec un maximum moyen ambiant de $40^{\circ} \mathrm{C}$ et un minimum moyen ambiant de $26^{\circ} \mathrm{C}$.

Durant cette période l'insolation n'est pas toujours importante les aérosols et la brume de sable étant abondants.

Nous pouvons, à partir d'expériences faites sur place, retenir pour :

$\begin{array}{lrrrr}T_{\mathrm{mb}} & 20^{\circ} & 25^{\circ} & 30^{\circ} \mathrm{C} & \\ T_{\mathrm{me}} & -10^{\circ} & 0^{\circ} & & \\ T_{\mathrm{Mb}} & 90^{\circ} & 100^{\circ} & 120^{\circ} & 140^{\circ} \mathrm{C} \\ T_{\mathrm{Mc}} & 30^{\circ} & 50^{\circ} & & \end{array}$

4.2 RÉSUltats POSSIBles A PARTIR DE BINAIRES LIQUIDES. - Les conditions d'exploitation des diagrammes 1 et 2 étant définies nous suivons le processus indiqué pour connaître les concentrations extrêmes qu'elles permettent d'envisager à l'équilibre. Pour rester lisible, la figure 3 ne retient qu'une température au condenseur durant le jour $T_{\mathrm{MC}}$ soit $50^{\circ} \mathrm{C}$ - partie inférieure : solutions pauvres - et une température à l'évaporateur durant la nuit soit $T_{\mathrm{me}}-10^{\circ} \mathrm{C}-$ partie supérieure : solutions riches.

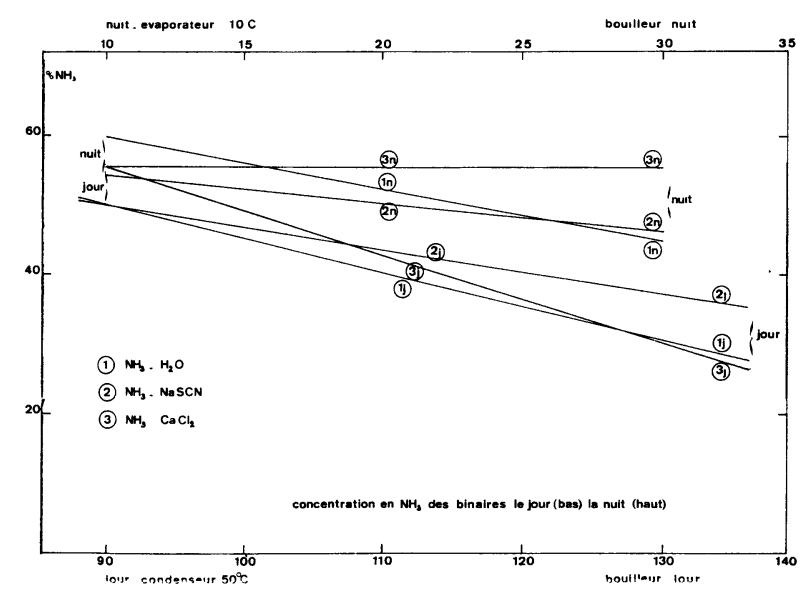

Fig. 3. - Concentration des mélanges binaires dans les conditions d'emploi

$1 \mathrm{H}_{2} \mathrm{O}, \mathrm{NH}_{3} \quad 2 \mathrm{NaSCN}, \mathrm{NH}_{3}$.

[Concentration of the binary mixtures in the employed conditions $1 \mathrm{H}_{2} \mathrm{O}, \mathrm{NH}_{3} \quad 2 \mathrm{NaSCN}, \mathrm{NH}_{3}$.]

On constate que si le bouilleur ne peut dépasser $90^{\circ} \mathrm{C}$ en saison chaude aucun des deux couples frigorigènes ne convient pour obtenir une capacité de réfrigération utilisable. Nous appelons capacité de réfrigération $Q=M C\left(\theta_{\mathrm{amb}}-\theta_{\mathrm{me}}\right)$ le nombre de frigories nécessaire à l'abaissement de température $\left(\theta_{\mathrm{amb}}-\theta_{\mathrm{me}}\right)$ d'une masse $M$ de nourriture, de chaleur massique $C$, placée dans le compartiment froid de l'appareil afin de la conserver. C'est le problème 
inverse de celui rencontré dans les zones tempérées en hiver [9] où l'énergie rayonnante est trop faible pour que la température d'un collecteur plan soit suffisante pour permettre le chauffage domestique.

Il faut, d'une part, un rayonnement solaire assez intense pour dépasser largement $100^{\circ} \mathrm{C}$ [3] et une température diurne non excessive pour obtenir une liquéfaction d'ammoniac importante; d'autre part, une température nocturne assez basse pour permettre une absorption rapide dans une solution suffisamment concentrée. Par ailleurs toujours sur la figure 3 (p.rtie basse : jour), la comparaison des pentes relatives aux mélanges (1) $\mathrm{NH}_{3}, \mathrm{H}_{2} \mathrm{O}$ et (2) $\mathrm{NH}_{3}$, NaSCN montre immédiatement que, malgré l'inconvénient de l'entraînement de l'eau par l'ammoniac au cours de sa vaporisation et l'avantage apparent de la faible tension de vapeur de $\mathrm{NaSCN}$, le mélange 1 reste préférable au mélange 2 aux températures élevées puisque, dans les mêmes conditions, pour une même élévation de température du couple dans le bouilleur, la solution $\mathrm{NH}_{3}-\mathrm{H}_{2} \mathrm{O}$ est la plus pauvre. Inversement sur la même figure (partie haute : nuit), dans la même ambiance, la solution $\mathrm{NH}_{3}-\mathrm{H}_{2} \mathrm{O}$ est la plus riche jusqu'à une certaine température.

Cette conclusion est conforme à celle de A. EggersLura et al. [6] lorsqu'il définit les coefficients de performance des 2 systèmes. Le travail expérimental de Swartman et al. [5] conduit à des résultats différents. Si nous en recherchons les causes, nous observons que les diagrammes de Swartman correspondent à des expériences où l'ambiante nocturne est en moyenne de $10^{\circ} \mathrm{C}$ et où la température diurne de la solution varie entre $30^{\circ} \mathrm{C}$ et $90^{\circ} \mathrm{C}$. Il s'agit alors de conditions absolument différentes de celles que nous nous sommes imposées. D'autre part, la figure 3 (jour) montre l'existence d'une intersection à $90^{\circ} \mathrm{C}$ (condenseur à $50^{\circ} \mathrm{C}$ ), des droites donnant la concentration des solutions ce qui signifie qu'en dessous de cette température, les effets sont inversés : la solution chaude de $\mathrm{NH}_{3}-\mathrm{NaSCN}$ est alors la plus pauvre donc permettra d'obtenir la plus grande masse d'ammoniac lorsque l'ambiante $<25^{\circ} \mathrm{C}$.

4.3 ETUDE QUANTITATIVE SUR UN EXEMPLE. - La figure 6 obtenue à partir de la précédente, visualise les mêmes remarques et leur donne une signification quantitative. Nous observons en particulier comment au-dessus de $90^{\circ} \mathrm{C}$ dans une ambiance tropicale $\left(\theta\right.$ ambiante nuit $30^{\circ} \mathrm{C}, \theta$ condenseur jour $\left.50^{\circ} \mathrm{C}\right)$ l'absorption sèche apparaît comme la plus rentable. Fixons les températures :

$T_{\mathrm{mb}}=30^{\circ} \mathrm{C}, \quad T_{\mathrm{me}}=-10^{\circ} \mathrm{C}$,

$T_{\mathrm{Mb}}=110^{\circ} \mathrm{C}, \quad T_{\mathrm{Mc}}=40^{\circ} \mathrm{C}$

et supposons que nous disposions initialement de $2 \mathrm{~kg} 500$ d'eau et $2 \mathrm{~kg} 500$ de NaSCN.

La figure 3 et un calcul immédiat nous permettent de définir dans les 2 cas : $m_{0}:$ masse totale initiale du mélange riche, $m_{1}$ : masse initiale d'ammoniac dans le mélange, $m_{2}$ : masse d'ammoniac liquéfié dans les conditions imposées.

$\begin{array}{lcc} & \mathrm{NH}_{3}, \mathrm{H}_{2} \mathrm{O} & \mathrm{NH}_{3}, \mathrm{NaSCN} \\ C_{0} \% & 45 & 44 \\ C_{1} \% & 36 & 39,5 \\ m_{0} \mathrm{~kg} & 4,5 & \simeq 4,5 \\ m_{1} \mathrm{~kg} & 2 & \simeq 2 \\ m_{2} \mathrm{~kg} & 0,6 & 0,36\end{array}$

En effet dans le cas $\left(\mathrm{NH}_{3}, \mathrm{H}_{2} \mathrm{O}\right)$ soit $x$ la masse en $\mathrm{kg}$ d'ammoniac initialement dissoute dans l'eau :

$0,45=\frac{x}{x+2,5}, \quad x=2,04 \simeq 2 \mathrm{~kg}$

soit $y$ la masse en $\mathrm{kg}$ d'ammoniac dans la solution pauvre :

$0,35=\frac{y}{y+2,5}, \quad y=1,35 \simeq 1,4 \mathrm{~kg} ;$

la masse d'ammoniac vaporisé est donc environ $0,6 \mathrm{~kg}$. Un calcul analogue conduit à $0,36 \mathrm{~kg}$ si l'on utilise le binaire $\mathrm{NaSCN}, \mathrm{NH}_{3}$.

Ainsi, en imposant aux 2 binaires des conditions identiques on peut prévoir à priori que l'un est mieux adapté que l'autre à la réfrigération. Ajoutons, pour être complet, que la tension de vapeur de l'eau nécessite l'emploi d'un réfrigérant ascendant qui n'est plus nécessaire avec le sulfocyanure de sodium, sel dont la tension de vapeur est négligeable, mais les seules données thermodynamiques sont favorables à l'emploi de l'eau dans le cas d'une ambiance tropicale.

Si nous désirons traduire, dans l'exemple cité, les conclusions en frigories nous notons que l'enthalpie de $\mathrm{NH}_{3}$ gaz à $40^{\circ} \mathrm{C}$ [7] est par kilogramme : $H_{\text {gaz }}=408,37 \mathrm{kcal}$, celle de $\mathrm{NH}_{3}$ liquide

$H_{\text {liquide }}=145,52$

ce qui entraîne une chaleur de vaporisation de $L_{\left(40{ }^{\circ} \mathrm{C}\right)}=262,85 \mathrm{kcal} / \mathrm{kg}$. A $-10^{\circ} \mathrm{C}$ nous trouvons respectivement $H_{\mathrm{gaz}}=398,67 ; H_{\text {liquide }}=89,03$ soit $L_{\left(-10^{\circ} \mathrm{C}\right)}=309,64 \mathrm{kcal} / \mathrm{kg}$ mais il faut refroidir le liquide $\mathrm{NH}_{3}$ de $40^{\circ} \mathrm{C}$ à $-10^{\circ} \mathrm{C}$ et comme les fuites thermiques augmentent avec l'écart à l'ambiante ce n'est guère plus de 200 kilofrigories nettes qui seront disponibles soit dans l'exemple étudié $120 \mathrm{kcal}$ dans le cas de l'eau et $72 \mathrm{kcal}$ avec le sulfocyanure de sodium. Ceci, sans tenir compte de l'ensemble de la capacité calorifique de l'évaporateur vide. Au moins en ambiance chaude, NaSCN semble donc inadapté à un tel usage malgré des caractères apparemment favorables [5] tels que sa faible volatilité et son absence d'agressivité vis-à-vis des métaux.

5. Processus chimique en phase solide : les ammoniacates. - 5.1 GÉNÉRALITÉs. - C'est surtout en Allemagne entre les 2 guerres qu'ont été examinées 
les propriétés absorbantes des sels alcalino-terreux. En particulier Plank [10], Linge [11], Altenkirsch [12], Mehl [13] ont contribué à la réalisation d'armoires ménagères à cycle intermittent d'absorption par voie sèche.

Par ailleurs E. Bonauguri [17] expérimentait en laboratoire à Milan de 1963 à 1965 le couple $\mathrm{NH}_{3}-\mathrm{CaCl}_{2}$ et annonçait des performances flatteuses de 23 à $70 \%$. Sur le même binaire, en Russie D. Muradov et O. Shadiev [18] travaillant à Tashkent en ambiance torride (jour $42^{\circ} \mathrm{C}$, nuit $19^{\circ} \mathrm{C}$ ) maintenaient l'évaporateur à $8{ }^{\circ} \mathrm{C}$ le jour, puis réalisaient [19] un appareil à glace libérant $400 \mathrm{kcal} / \mathrm{m}^{2} /$ jour ; Shadiev et Umarov [20] examinaient les problèmes de conductivité et l'importance des fuites thermiques. Dans l'octoammoniacate la conductivité thermique variait de $0,65 \mathrm{kcal} / \mathrm{hm}{ }^{\circ} \mathrm{C}$ pour un écart de $17^{\circ} \mathrm{C}$ à $2,45 \mathrm{kcal} / \mathrm{hm}{ }^{\circ} \mathrm{C}$ pour une différence de température de $37^{\circ} \mathrm{C}$.

L'utilisation pratique durant des années d'appareils domestiques reposant sur ce principe est rassurante, la question essentielle est donc de définir comment la distribution du rayonnement solaire et les performances qu'il impose sont compatibles avec une exploitation réelle du froid solaire.

D'après Eggers-Lura [6] les 2 sels les mieux adaptés à cet objet sont le chlorure de calcium $\mathrm{CaCl}_{2}$ et le chlorure de strontium $\mathrm{SrCl}_{2}$ dont les performances d'absorption de $\mathrm{NH}_{3}$ sont équivalentes.

Si l'on tient compte du fait que $\mathrm{CaCl}_{2}$ est un sousproduit actuellement inutilisable de l'industrie Solvay et de la parenté chimique de $\mathrm{Ca}$ et $\mathrm{Sr}$ on peut se limiter dans une première approche au seul $\mathrm{CaCl}_{2}$.

5.2 DisSOCIATION ET RÉGÉNÉRATION DE L'OCTOAMMONIACATE. - L'octoammoniacate $\mathrm{CaCl}_{2}, 8 \mathrm{NH}_{3}$ se forme à l'ambiante. Par chauffage on obtient une dissociation progressive suivant le schéma [14]

$\mathrm{CaCl}_{2}, 8 \mathrm{NH}_{3} \underset{95^{\circ}}{\longrightarrow} \mathrm{CaCl}_{2}, 4 \mathrm{NH}_{3} \underset{106^{\circ}}{\longrightarrow} \mathrm{CaCl}_{2}, 2 \mathrm{NH}_{3}$ $p(=16 \mathrm{~atm})+.4 \mathrm{NH}_{3}, \quad p(=16 \mathrm{~atm})+.2 \mathrm{NH}_{3}$

(condenseur à $40^{\circ}$ )

ce qui libère $6 \mathrm{NH}_{3}$ par mole $\mathrm{CaCl}_{2}$ initiale lorsque les conditions imposées sont celles définies plus haut. La séparation des $2 \mathrm{NH}_{3}$ restants n'est possible qu'au-delà de $180^{\circ} \mathrm{C}$ [14]. Il ne peut être question de l'envisager pour la réfrigération employant le rayonnement solaire non concentré.

En fait la température caractérisant ces réactions est largement dépendante de la pression subie par les divers ammoniacates. Par exemple [14] l'octoammoniacate devient le tétraammoniacate à

$95^{\circ} \mathrm{C}$ si $p=16$ bars

$32{ }^{\circ} \mathrm{C}$ si $p=1$ bar

$-2{ }^{\circ} \mathrm{C}$ si $p=1 / 7,6$ bar

le tétraammoniacate forme le diammoniacate à

$106^{\circ} \mathrm{C}$ sous 16 bars

$42{ }^{\circ} \mathrm{C}$ sous 1 bar

$7^{\circ} \mathrm{C}$ sous $1 / 7,6$ bar.
D’autre part la vitesse de dissociation dépend également de la température et de la quantité d'ammoniacate présent dans le mélange solide. Dans des conditions données, plus la quantité d'ammoniacate diminue, plus la transformation est lente. Or, nous sommes limités, à la régénération, par les 17 heures séparant la fin du chauffage par le soleil au début de l'échauffement du jour suivant.

En se limitant à un strict bilan réactionnel, au total $102 \mathrm{~g}$ d'ammoniac sont utilisables par $111 \mathrm{~g}$ de chlorure de calcium. Si nous nous référons à la comparaison vue en absorption humide, à $2,5 \mathrm{~kg}$ de $\mathrm{CaCl}_{2}$ correspondent $2,3 \mathrm{~kg}$ d'ammoniac récupérable, donc en tenant compte de la dissociation précédente ce sont $460 \mathrm{kcal}$ brutes qui caractérisent le pouvoir réfrigérant du sel, soit 4 fois plus qu'avec le binaire eau- $\mathrm{NH}_{3}$.

Notons que ces résultats n'ont de sens qu'à l'équilibre, aussi bien pour le liquide absorbant que pour le solide. En fait, comme nous l'avons déjà signalé, le paramètre essentiel demeure la vitesse d'absorption de l'ammoniac au cours de la nuit.

Aussi, dans la réalité, les réactions ne sont jamais complètes, soit en raison de la température diurne au collecteur, soit faute de temps pour la régénération de l'ammoniacate. Il n'est pas inutile sur ce point de consulter des ouvrages pratiques. Degoin [15] considère que la saturation à froid est à peu près terminée avec $600 \mathrm{~g}$ de $\mathrm{NH}_{3}$ par kilo de $\mathrm{CaCl}_{2}$ qui retient à chaud environ $200 \mathrm{~g}$ de $\mathrm{NH}_{3}$, donc $400 \mathrm{~g}$ seulement de réfrigérant sont disponibles par kilo de sel anhydre, ce qui représente $1 \mathrm{~kg}$ d'ammoniac dans l'exemple étudié, très supérieur à ce que laisse espérer le meilleur des binaires liquides à l'équilibre.

Le même auteur évalue à 1200 frigories la capacité frigorifique par 24 heures d'une armoire domestique, ce qui, d'après les données, requiert l'emploi d'un minimum de $15 \mathrm{~kg}$ de $\mathrm{CaCl}_{2}$ car il convient de tenir compte des fuites thermiques et de la capacité calorifique particulière de l'appareil. D'après nos propres études [2] c'est environ $4 \mathrm{~m}^{2}$ d'insolateurs plans qui seraient nécessaires pour chauffer le sel dans des temps compatibles avec la durée d'ensoleillement efficace.

Ces propositions, issues de l'expérience courante, montrent comment dans les conditions d'utilisation réelles la vitesse de dissociation correspond à la libération des $2 / 3$ de la quantité d'ammoniac théoriquement disponible durant la période de chauffage et l'entrée en combinaison des $2 / 3$ de cette masse pendant la nuit soit une performance possible de $43 \%$.

Il est bien évident que les températures maximales et minimales réelles du générateur peuvent modifier ce taux moyen d'utilisation.

5.3 ETUdE PHYSICO-CHIMIQUE AU LABORATOIRE. Il serait tentant de comparer ces données physicochimiques fondamentales à celles que nous pourrions obtenir expérimentalement dans une perspective définie par les diagrammes $(\log P, 1 / T)$. Nous avons donc 
construit au laboratoire un ensemble permettant d'étudier ces réseaux pour les couples frigorigènes les plus variés et d'abord le couple $\mathrm{NH}_{3}-\mathrm{CaCl}_{2}$.

5.3.1 L'appareil : figure 4. - Pour des raisons de précision et de validité des mesures, il convenait de travailler sur des masses suffisantes. Or d'après Plank-Kuprianoff [10] le gonflement considérable de l'ammoniacate exige un volume libre de $486 \mathrm{l} /$ mole de sel anhydre soit $4,4 \mathrm{dm}^{3} / \mathrm{kg}$ ce qui, compte tenu de sa densité $(2,15)$, exige $9,5 \mathrm{dm}^{3}$ par $\mathrm{dm}^{3}$ donc près de 10 fois son volume. Nous avons donc retenu $2 \mathrm{~kg}$ de sel anhydre disposé de manière à augmenter au maximum l'interface solide-gaz dans une enceinte d'acier à l'épreuve de 30 bars, entourée de rubans chauffants permettant de travailler en température contrôlée à $\pm 0,5^{\circ} \mathrm{C}$ près, de l'ambiante à $150^{\circ} \mathrm{C}$. Des manomètres assurent la mesure des pressions. Le bouilleur est réuni par l'intermédiaire d'un condenseur à un évaporateur de $5 \mathrm{dm}^{3}$ environ muni d'un tube de verre à l'épreuve de la pression et qui permet des lectures de volume de liquide à $10 \mathrm{~cm}^{3}$ près.

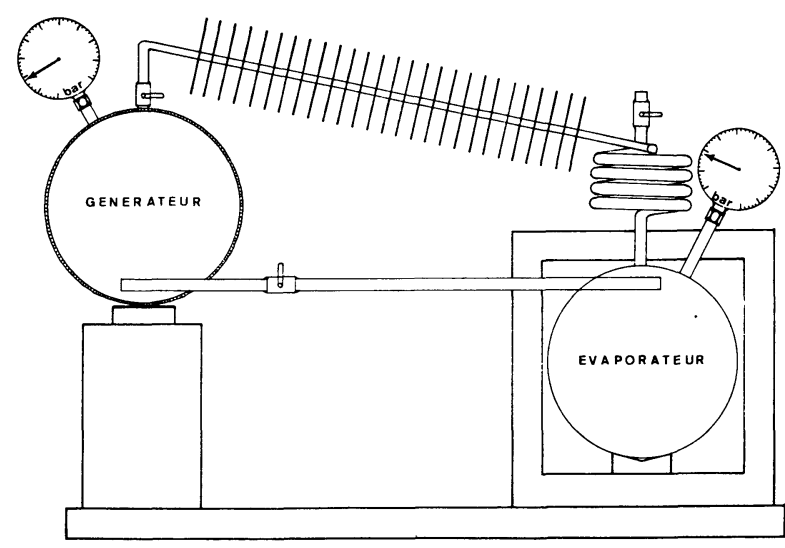

apparell pour l'etude des equillibres $\mathrm{NH}_{3} \mathrm{CaCh}$

Fig. 4. - Appareil pour l'étude des équilibres $\mathrm{NH}_{3}-\mathrm{CaCl}_{2}$.

[Apparatus for the study of the equilibriums $\mathrm{NH}_{3}-\mathrm{CaCl}_{2}$.]

Après de délicats essais d'étanchéité à l'azote sous 30 bars et une mise sous vide prolongée de l'appareil renfermant $\mathrm{CaCl}_{2}$ anhydre, nous introduisons $2,7 \mathrm{~kg}$ d'ammoniac liquide dans l'évaporateur. Un jeu de vannes permet de réaliser dans le bouilleur tous les taux d'ammoniacate permis par la stœchiométrie.

5.3.2 Première série d'expériences. - Soit une concentration $C$ du couple solide gaz isolé dans le bouilleur. A l'aide du thermomètre régulateur Jumo noyé dans la masse, on porte l'ensemble à une température $T$ et on note à intervalles réguliers la pression afin de la définir ainsi que sa vitesse de mise en équilibre. Même si une partie de l'ammoniacate se dissocie durant cette opération, la composition de la phase pâteuse varie peu. En effet, le volume libre dans le générateur n'excède pas $5 \mathrm{l}$, qui, même sous $15 \mathrm{~kg}$ de pression ne correspondent pas une masse supérieure à $50 \mathrm{~g}$ [7].

La précision sur la concentration lue au départ peut donc être au maximum de $5 \%$ en milieu pauvre en ammoniac à $1 \%$ en milieu riche.

Nous pouvons ainsi tracer dans le diagramme $(\log P, 1 / T)$ le réseau des droites correspondant à des concentrations variant de 25 à $55 \%$ d'ammoniac.

5.3.3 Résultats dans les conditions d'équilibre. Ils sont portés sur la figure 5 . Ils permettent d'apprécier sur l'exemple retenu en 5.3 la validité des conclusions issues du raisonnement purement chimique de 5.2 sur la dissociation et la régénération de l'octoammoniacate.

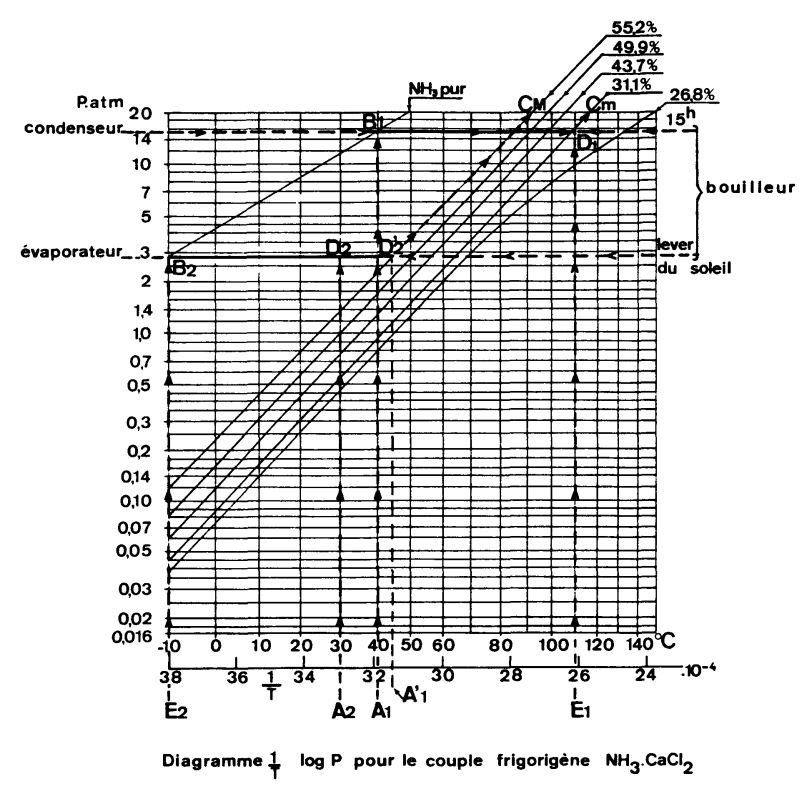

Fig. 5. - Diagramme 1/T, Log $P$ pour le couple $\mathrm{NH}_{3}-\mathrm{CaCl}_{2}$.

[Diagram $1 / T, \log P$ for the couple $\mathrm{NH}_{3}-\mathrm{CaCl}_{2}$.]

En effet pour $T_{\mathrm{MC}}=40^{\circ} \mathrm{C}$ (point $\mathrm{A}_{1}$ ), nous trouvons, à la verticale de $A_{1}$, la pression définie par $B_{1}$ sur la droite $\mathrm{NH}_{3}$ pur soit 16 bars, l'horizontale issue de $\mathrm{B}_{1}$ coupe en $\mathrm{D}_{1}$ la droite $\mathrm{C}_{\mathrm{m}}=30 \%$ à la verticale de $\mathrm{E}_{1}\left(T_{\mathrm{mb}}=110^{\circ} \mathrm{C}\right.$ ). Si $T_{\mathrm{me}}=-10^{\circ} \mathrm{C}$ (point $\mathrm{E}_{2}$ ) la verticale issue de $\mathrm{E}_{2}$ coupe la droite $\mathrm{NH}_{3}$ pur en $\mathrm{B}_{2}$ soit 2,9 bars. De $\mathrm{B}_{2}$ l'horizontale coupe la droite $C_{\mathrm{M}}=55,7 \%$ en 1 point $\mathrm{D}_{2}^{\prime}$ dont l'abscisse est $\mathrm{A}_{1}^{\prime}$ $\left(44^{\circ} \mathrm{C}\right)$ or $\mathrm{D}_{2}\left(T_{\mathrm{mb}}=30^{\circ} \mathrm{C}\right)$ a pour abscisse $\mathrm{A}_{2}$ ce qui signifie que $C_{\mathrm{M}}=55,7 \%$ est le maximum possible de la quantité d'ammoniac présente dans l'ammoniacate.

Or nous pouvons comparer les résultats en raisonnant sur 2,500 $\mathrm{kg} \mathrm{de} \mathrm{CaCl}_{2}$.

Soit :

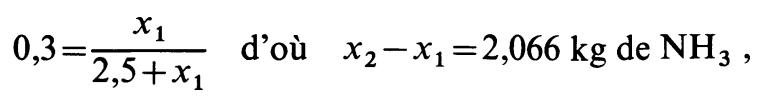

$0,557=\frac{x_{2}}{2,5+x_{2}}$ disponibles pour la réfrigération 
La stœchiométrie nous avait conduit à $2,300 \mathrm{~kg}$. L'écart observé tient au fait qu'à $110^{\circ} \mathrm{C}$ nous n'avons pas atteint le diammoniacate. Il faudra chauffer au-delà de cette température si nous voulons libérer le maximum de liquide frigorigène.

6. Comparaison des 3 couples frigorigènes. 6.1 Diagramme D'éQuilibre (P, T). - A partir de l'expérience, nous vérifions tout l'intérêt que présente, en zone tropicale, l'emploi d'un absorbant sec. Nous généralisons ces résultats sur la figure 3, en notant, pour les 3 couples étudiés, lorsque le condenseur est à $50^{\circ} \mathrm{C}$ le jour et l'évaporateur à $-10^{\circ} \mathrm{C}$ la nuit, la concentration en ammoniac en fonction de la température du bouilleur chaud le jour, froid la nuit. La figure 6 montre clairement les possibilités et les limites des 3 binaires à condition de travailler en ambiance tropicale avec un collecteur plan permettant de dépasser largement $100^{\circ} \mathrm{C}$ aux heures les plus chaudes. Sous cette réserve, $\Delta C \%$ de liquide frigorigène utilisable avec $\mathrm{NH}_{3}-\mathrm{CaCl}_{2}$ est environ 2 fois la valeur obtenue avec $\mathrm{NH}_{3}-\mathrm{H}_{2} \mathrm{O}$ et 4 fois celle définie par $\mathrm{NH}_{3}-\mathrm{NaSCN}$.

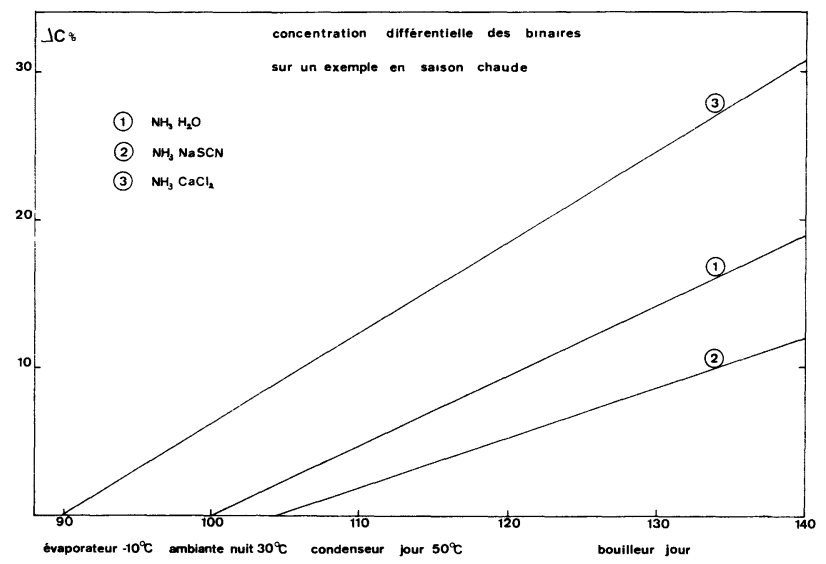

Fig. 6. - Fraction d'ammoniac utilisable dans les conditions extrêmes.

[Fraction of ammonia available in the extreme conditions.]

6.2 Optimisation Des DiAgRAMMES EN VUE DE LA RÉFriGÉRATION SOLAIRE. - Nous pouvons nous demander, au vu de ces résultats, quels caractères doivent présenter les diagrammes des couples les mieux adaptés à la réfrigération solaire. En comparant les figures $1-2$ et 5 nous constatons que, dans le domaine des concentrations utilisables limité par $25 \%$ et $55 \%$, le réseau du binaire le plus rentable est le plus étroit. Ce qui signifie que l'écart de température le plus faible permet la plus grande variation de concentration. C'est bien le cas de $\mathrm{NH}_{3}-\mathrm{CaCl}_{2}$.

D'autre part, plus la pente des droites $(\log P, 1 / T)$ est grande, plus la pression augmente lorsque la température s'élève, ce qui assure une bonne distillation de l'ammoniac, plus la pression diminue lorsque la température s'abaisse, ce qui permet une évaporation rapide de l'ammoniac ainsi qu'une pression limite plus basse, donc une plus faible température limite à l'évaporateur. La figure 7 montre comment, aux concentrations moyennes, les pentes respectives des trois droites répondent à cette exigence. Cette propriété demeure aux concentrations extrêmes. Nous recherchons les basses pressions en fin de nuit, les hautes pressions en fin de journée, avec un écart maximum des concentrations.

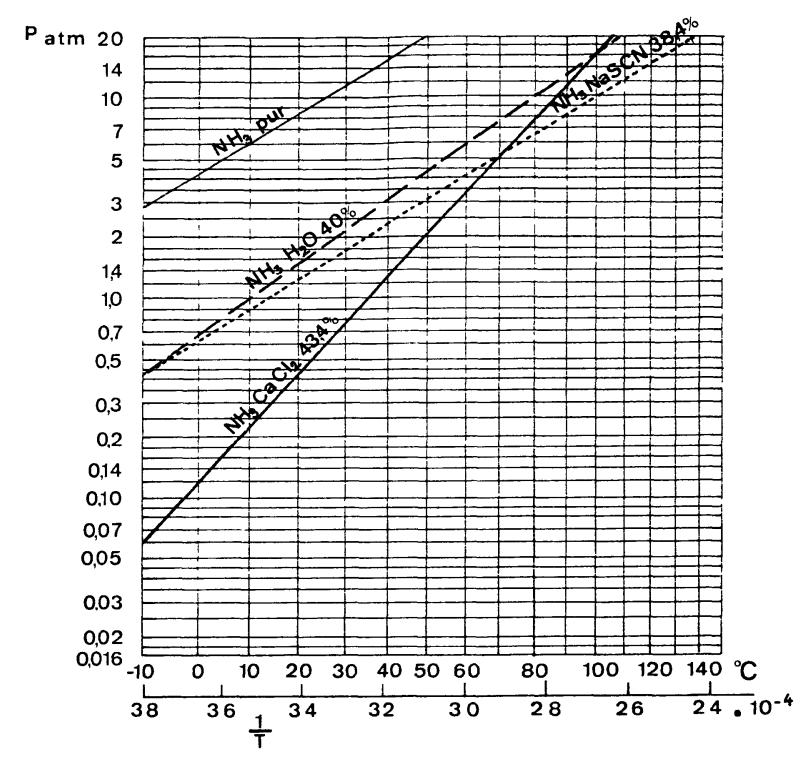

Fig. 7. - Pentes comparées des droites $1 / T, \log P$ des 3 couples frigorigènes aux moyennes concentrations.

[Comparated slopes of the straight lines $(1 / T, \log P)$ of the 3 frigorigene couples at the medium concentrations.]

7. Considérations cinétiques dans le cas du couple $\mathrm{NH}_{3}-\mathrm{CaCl}_{2}$. - Nous avons indiqué l'importance de la cinétique des transformations. Nous sommes en effet limités à un maximum de 6 heures pour la distillation, de 18 heures pour la régénération. Ce sont donc les débuts de courbe représentant les vitesses qui présentent de l'intérêt.

Deux types d'évolution isotherme caractérisant le comportement du binaire :

- la vitesse de condensation de $\mathrm{NH}_{3}$,

- la vitesse d'approche de l'équilibre du couple isolé.

7.1 Condensation. - Les variables sont $P, T, C$ et $v=\mathrm{d} m / \mathrm{d} t$ masse d'ammoniac condensé par unité de temps. La figure 8 précise à partir de diverses concentrations initiales, sous une pression fixe, à une température donnée, le volume d'ammoniac libéré par heure et le volume horaire moyen durant un chauffage de $5 \mathrm{~h}$ (durée journalière exploitable du rayonnement solaire). 


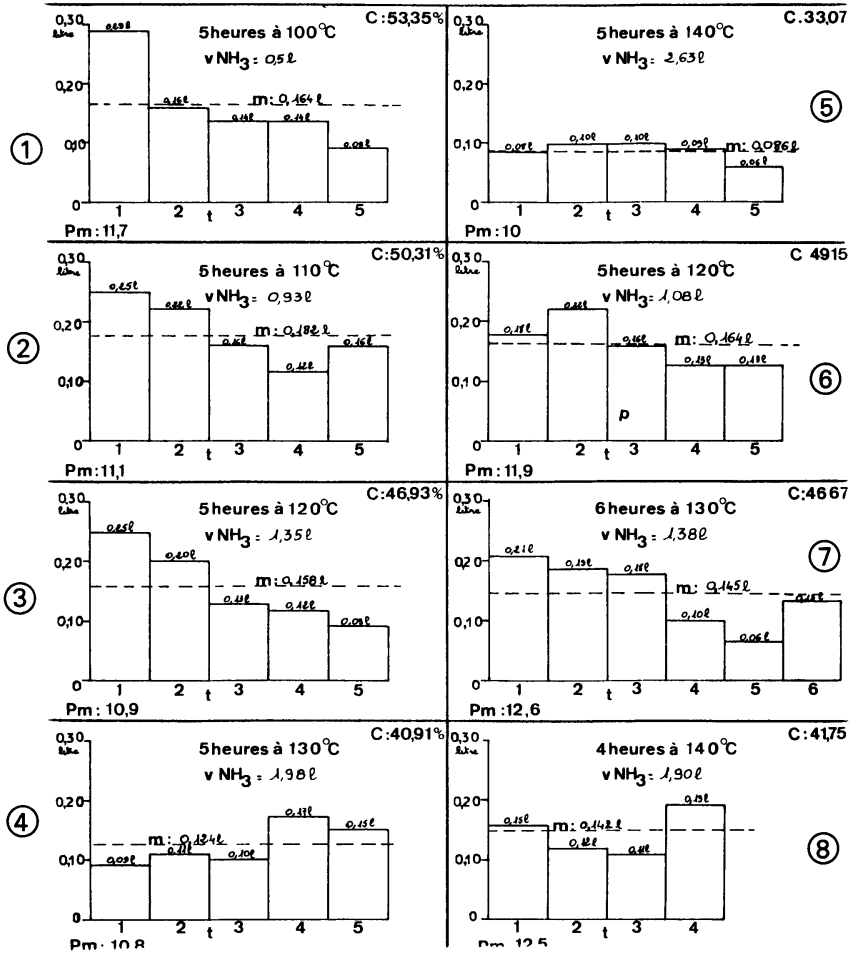

Fig. 8. - Vitesse horaire de libération de $\mathrm{NH}_{3}$ dans des conditions données.

$C$ : concentration $\mathrm{NH}_{3}$

$v \mathrm{NH}_{3}: \mathrm{NH}_{3}$ en litres au départ dans l'évaporateur

$P_{\mathrm{m}}$ : pression moyenne en bars

$t:$ temps en heures

$m:$ moyenne.

[Speed per hour of liberation of $\mathrm{NH}_{3}$ in know conditions.]

Numérotons les expériences de 1 à 8 . Nous en tirons les observations qualitatives suivantes :

1) plus l'ammoniacate est pauvre, plus la vitesse moyenne $v_{\mathrm{m}}$ de condensation comptée sur $5 \mathrm{~h}$ diminue, à $T$ et $P$ fixes, cette propriété persiste même si l'on élève la température $T$ (expériences 3 et 6 );

2) à concentration $C$ et pression $P$ fixes, la vitesse $v_{\mathrm{m}}$ croît lorsqu'on élève la température (expériences 4 et 8 ). Si l'on compare 3 à $7, v_{\mathrm{m}}$ décroît mais la pression $P_{3}<P_{7}$. De même de 2 à $6 v_{\mathrm{m}}$ décroît mais $C_{6}<C_{2}$ et $P_{6}>P_{2}$;

3) à $T$ et $C$ fixes la vitesse $v_{\mathrm{m}}$ croît lorsqu'on abaisse la pression (expériences 3 et 7 où $v_{\mathrm{m} 3}>v_{\mathrm{m} 7}$ malgré l'augmentation de température).
Nous pouvons traduire cela quantitativement en partant de $f_{1}$ : fraction d'ammoniac restant à libérer au début de l'expérience et de $f_{2}$ : fraction d'ammoniac libérée en 5 heures.

Tableau I.

\begin{tabular}{|c|c|c|c|c|c|}
\hline$\theta^{\circ} \mathrm{C}$ & 100 & 110 & 120 & 130 & 140 \\
\hline$f_{1}$ & 0,84 & 0,71 & $0,58 \quad 0,66$ & $0,39 \quad 0,57$ & $0,19 \quad 0,41$ \\
\hline & 0,31 & 0,39 & $0,41 \quad 0,37$ & $0,48 \quad 0,39$ & $0,69 \quad 0,42(41$ \\
\hline$P$ bars & 11,7 & 11,1 & $10,9 \quad 11,9$ & $10,8 \quad 12,6$ & $10 \quad 12,5$ \\
\hline
\end{tabular}

Précisons, par exemple sur l'expérience 1, comment nous obtenons $f_{1}$ et $f_{2}$. Nous travaillons sur $2 \mathrm{~kg}$ de $\mathrm{CaCl}_{2}$ et $2,47 \mathrm{~kg}$ de $\mathrm{NH}_{3}$ qui, après formation de la combinaison $\mathrm{CaCl}_{2}, 8 \mathrm{NH}_{3}$, peuvent libérer $1,85 \mathrm{~kg}$ de $\mathrm{NH}_{3}$ soit, 1,86/0,61 = 3,1 1 de $\mathrm{NH}_{3}$ (densité de $\mathrm{NH}_{3}$ liquide $=0,61$ dans les conditions d'emploi).

Si au départ $\mathrm{C}: 53,35 \%$, l'évaporateur contient 0,51 on pourrait donc évaporer 2,6 1 de $\mathrm{NH}_{3}$ soit $f_{1}=2,6 / 3,1=0,84$ (cf. : tableau I).

$\mathrm{Au}$ bout de 5 heures, on a recueilli l'équivalent de $0,1641 \times 5=0,8201$ de $\mathrm{NH}_{3}$ soit $f_{2}=0,82 / 2,6=0,31$ (cf. : tableau I). Nous vérifions immédiatement sur ce tableau la validité des observations précédentes.

Notons que $f_{1}+f_{2} \neq 1$ puisqu'on se réfère à des quantités de $\mathrm{NH}_{3}$ différentes : l'une, en début d'expérience, se référant à la totalité de $\mathrm{NH}_{3}$ récupérable, l'autre à la quantité de $\mathrm{NH}_{3}$ réellement disponible. Cette distinction a été rendue nécessaire dans le but de travailler sur des concentrations variées : riche, moyenne, pauvre, et en vue de se conformer aux futures conditions d'emploi, les exigences climatiques quotidiennes nous interdisant une référence fixe pour le système en début de journée.

7.2 VITESSE D'APPROCHE DE L'ÉQUILIBRE. - Le système biphasé $\mathrm{NH}_{3}-\mathrm{CaCl}_{2}$ étant isolé, pour établir le diagramme $(1 / T, \log P)$ nous notons, à diverses températures maintenues constantes, pour une même concentration en ammoniac, l'évolution de la pression en fonction du temps.

Les premiers résultats sont consignés dans le tableau II où nous portons pour chaque température et chaque composition la pression d'équilibre $P$ en bars et la vitesse moyenne relative, prise sur les

Tableau II.

\begin{tabular}{|c|c|c|c|c|c|c|c|c|c|c|c|c|}
\hline \multirow[b]{2}{*}{$C \%$} & \multicolumn{2}{|c|}{$50^{\circ}$} & \multicolumn{2}{|c|}{$60^{\circ}$} & \multicolumn{2}{|c|}{$70^{\circ}$} & \multicolumn{2}{|c|}{$80^{\circ}$} & \multicolumn{2}{|c|}{$90^{\circ}$} & \multicolumn{2}{|c|}{$100^{\circ}$} \\
\hline & $P$ & $v_{\mathrm{m}}$ & $P$ & $v_{\mathrm{m}}$ & $P$ & $v_{\mathrm{m}}$ & $P$ & $v_{\mathrm{m}}$ & $P$ & $v_{\mathrm{m}}$ & $P$ & $v_{\mathrm{m}}$ \\
\hline$\overline{23,4}$ & & & 2 & 10 & & - & & & & & 63 & \\
\hline 26,8 & & & & & & & 4,5 & 11,4 & & & & \\
\hline 31,1 & 1,5 & 6,6 & 2,5 & 8 & & & 5,5 & 16,3 & & & & \\
\hline 36,8 & 2,3 & 4,3 & & & 5,4 & 8,3 & & & 10,8 & 12,8 & 14,6 & 6,1 \\
\hline 43,7 & & & 3,3 & 7,8 & 5,2 & 7,6 & 7,7 & 6,4 & 11,3 & 16,7 & 17,2 & 7,2 \\
\hline 49,9 & 2,8 & 5,1 & & & 6,8 & 19,1 & 10,7 & 7,8 & 15,7 & 10,1 & & \\
\hline 55,2 & 3,9 & 7,6 & 6 & 9 & 9 & 14,4 & & & 21 & 28,5 & & \\
\hline
\end{tabular}


2 premières heures $v_{\mathrm{m}}$ de l'évolution isotherme de la pression $v_{\mathrm{m}}=\frac{1}{P} \frac{\mathrm{d} P}{\mathrm{~d} t}$ en $\%$ par heure.

L'étude de ce phénomène ne présente qu'une apparente simplicité. En effet la variation de pression observée au passage entre 2 paliers de température correspond à 2 processus :

- la dilatation isochore du gaz,

- la dissociation ou la régénération de l'ammoniacate.

Or la vitesse de libération de $\mathrm{NH}_{3}$ au cours de la montée en température est largement supérieure à celle de son absorption si bien qu'il convient de décrire les courbes d'égale concentration dans les 2 sens en précisant, pour chaque température, et dans chaque sens, la cinétique de mise en équilibre. L'arrêt de l'absorption du gaz lié au comportement de l'interface peut se produire sous une pression légèrement plus forte que celle de son dégagement et après un temps qui peut atteindre une douzaine d'heures.

Nous avons rappelé que la pression modifie considérablement la température de dissociation des ammoniacates. Le tableau II montre en particulier qu'à toute concentration, au-delà de $100^{\circ} \mathrm{C}, v_{\mathrm{m}}$ reste faible : les dissociations sont largement entamées.

$\mathrm{Si}$ nous faisons correspondre les concentrations massiques $\mathrm{C} \%$ aux moles $\mathrm{m}$ de $\mathrm{NH}_{3}$ présentes par mole de $\mathrm{CaCl}_{2}$, soit :

$\begin{array}{lcccc}\mathrm{C} \% & 55,06 & 47,8 & 37,9 & 23,4 \\ m & 8 & 6 & 4 & 2\end{array}$

Nous notons que les concentrations élevées correspondent, entre 70 et $90^{\circ} \mathrm{C}$, aux vitesses les plus fortes. Le milieu étudié étant pâteux, les transferts de matière et de chaleur ne sont pas toujours repro- ductibles mais les ordres de grandeur sont compatibles : en dessous de $70^{\circ} \mathrm{C}$ les modifications relatives sont peu importantes. Cette évolution jouera un rôle en réfrigération.

8. Conclusion. - Nous avons examiné quelquesuns des aspects présentés par les couples frigorigènes susceptibles d'être utilisés à partir de l'énergie solaire.

Nous avons constaté que les conditions climatiques sont essentielles pour orienter le choix d'un couple déterminé. Si les températures nocturnes sont basses et le rayonnement direct intense, les absorbants secs liquides peuvent présenter de l'intérêt.

En zone tropicale où l'ambiance minimale est tiède et le rayonnement direct intense, les absorbants secs laissent espérer un rendement supérieur. A partir des réseaux de courbes d'égale concentration les performances annoncées sont des limites que réduiront les cinétiques réelles associées aux durées d'irradiation efficaces et de vaporisation génératrice de froid.

Cependant les comparaisons conduites dans des conditions identiques demeurent valables. Il convient simplement de poursuivre une étude systématique des phénomènes réels : importånce du gonflement et vitesse de formation des ammoniacates au cours de la régénération, risques de colmatage, concentrations riches et pauvres atteintes dans les délais imposés par l'intermittence des apports énergétiques.

La création d'interfaces solide-gaz étendues permettra d'accroître la vitesse d'absorption de $\mathrm{NH}_{3}$ donc la chute des températures à l'évaporateur.

Il conviendra probablement de s'orienter en réfrigération, comme cela s'impose dans les zones tempérées pour le chauffage, vers des ensembles mixtes où l'énergie solaire apparaît comme un appoint dont l'importance est conditionnée par le climat.

\section{Bibliographie}

[1] Flechon, J., Martin, G., Toure, I., C. R. Hebd. Séan. Acad. Sci. 280 B (1975) 211.

[2] Flechon, J., C. R. Hebd. Séan. Acad. Sci. 283 B (1976) 127.

[3] Flechon, J., Revue Phys. Appl. 11 (1976) 743-50.

[4] Flechon, J., Wertwijn, R. et Diallo, A., C. R. Hebd. Séan. Acad. Sci. 281 B (1975) 9.

[5] Swartman, R. K. et al., Colloque UNESCO, Paris, Juillet 1973, E 5 .

[6] EgGers-Lura, A. et al., Heliotechnique and Development (Ed. Kettani, Soussou) 1976, p. 95.

[7] Règles pour Machines Frigorifiques (Institut International du Froid, Paris) 5e ed. (1971) 114-15.

[8] George, C., Blytas, Farrington, Daniels, J. Amer. Chem. Soc. 84-71 (1962) 1075-83.

[9] FleChon, J., Journées Internationales sur le Chauffage Solaire. Liège, Sept. 1977.

[10] Plank, R. and KuPRianoff, J., Die Kleinkältemaschine (Spring Verlag, Berlin) 1960, p. 351.

[11] LINGE, K., Uber Periodische Absorptionskältemaschinen, D. Sc. Thesis Karlsruhe, May 1929.

[12] AltenKIRSCH, E., Absorptionshältemaschinen (Verlag Technik, Berlin) 1954.
[13] MeHL, W., Die Thermischen Eigenschaften Zeitschrift für die Gesamte Kälteindustrie (Heft 11) 1934, Vol. 41, p. 186

[14] Pascal, P., Traité de Chimie Minérale T (Masson) IV (1956-70) 316.

[15] Degorn, Réfrigération électrique automatique. Librairie Girardot (épuisé).

[16] Sargent, S. L. and Beckman, W. A., Theoritical performance of an Ammonia Sodium Thiocyanate intermittent absorption refrigeration cycle, Solar Energy 12 (1968) 137-146.

[17] Bonaugure, E., Preliminary notes on a Solar Absorption Unit, Proceedings of the 12th International Congress of Refrigeration, I.F.F., Paris (1969) 1411.

[18] Muradov, D. and Shadiev, O., "Intermittent solar refrigerator with solid absorbent ", Izd, Tash, G.V. i B.G.P.I., Tashkent (1969).

[19] Muradov, D. and Shadiev, O., " Testing of a Solar Refrigerator " Geliotekhnika 7 (1971) 33.

[20] Shadiev, O. and Umarov, G. Y., "On the temperature Distribution in a Solar Refrigerator Generator and the thermal conductivity of the Absorbent ", Geliotekhrika 8 (1974) 34. 